\title{
Lanreotide Depot: An Antineoplastic Treatment of Carcinoid or Neuroendocrine Tumors
}

\author{
Edward M. Wolin ${ }^{1} \cdot$ Amandine Manon $^{2} \cdot$ Christophe Chassaing $^{3} \cdot$ Andy Lewis $^{3}$. \\ Laurent Bertocchi $^{3} \cdot$ Joel Richard $^{3} \cdot$ Alexandria T. Phan ${ }^{4}$
}

Published online: 13 September 2016

(C) The Author(s) 2016. This article is published with open access at Springerlink.com

\begin{abstract}
Purpose Peptide drugs for antineoplastic therapies usually have low oral bioavailability and short in vivo half-lives, requiring less preferred delivery methods. Lanreotide depot is a sustained-release somatostatin analog (SSA) formulation produced via an innovative peptide self-assembly method. Lanreotide is approved in the USA and Europe to improve progression-free survival (PFS) in patients with unresectable gastroenteropancreatic neuroendocrine tumors (GEP-NETs) and also approved in Europe for symptom control in carcinoid syndrome associated with GEP-NETs. This review discusses how the distinct molecule and formulation of lanreotide depot provide advantages to patients and health care providers, as well as the most recent clinical evidence demonstrating the safety and efficacy of lanreotide depot in inhibiting tumor growth and controlling hormonal symptoms in GEP-NETs. Methodology and Results The lanreotide depot formulation confers a remarkable pharmacokinetic profile with no excipients, comprised only of lanreotide acetate and water. Of note, lanreotide depot constitutes an example for peptide selfassembly based formulations, providing insights that could help future development of sustained-release formulations of
\end{abstract}

Edward M. Wolin

ewolin@montefiore.org

1 Medical Oncology, Montifiore Einstein Center for Cancer Care, 1695 Eastchester Rd., Second Floor, Bronx, NY 10461, USA

2 Clinical Pharmacokinetics and Drug Metabolism, Ipsen Innovation, 5 Avenue du Canada, 91140 Les Ulis, France

3 Peptide Development, Beaufour Ipsen Industrie, Rue Ethe Virton, 28109 Dreux, France

4 Houston Methodist Hospital, 6410 Fannin St, Houston, TX 77030, USA other antineoplastic peptides. Most patients with GEP-NETs will present with inoperable or incurable disease; thus, medical management for symptoms and tumor control plays a crucial role. Recent long-term clinical studies have demonstrated that lanreotide depot is well tolerated, prolongs PFS in GEPNET patients, and significantly reduces symptoms related to carcinoid syndrome.

Conclusions The unique depot formulation and delivery method of lanreotide confer advantages in the treatment of metastatic GEP-NETs, contributing to improvements in NET-related symptoms and PFS without reducing quality of life in this patient population.

Keywords Lanreotide $\cdot$ Depot preparations $\cdot$ Neuroendocrine tumors $\cdot$ Drug formulation

\section{Introduction}

Peptides are attractive therapeutic molecules due to their significant activity and low toxicity profile. It is not surprising that the number of peptide-based drugs being evaluated in clinical trials has been steadily increasing over the years. Currently, many peptide drug candidates are in clinical or preclinical development phases, and many are being evaluated in the oncology setting [1]. However, peptide drugs usually have low and variable oral bioavailability and short in vivo half-lives. Therefore, they require delivery methods such as infusion, frequent injections, implantable devices, or intramuscular injection of large drug volumes, which are not always preferred by health care providers and patients $[2,3]$.

Fortunately, an intrinsic biophysical property of some peptides may help overcome the problems associated with peptide drug delivery. Under specific conditions of $\mathrm{pH}$, concentration, and salts, many peptides and proteins will self-assemble into 
filamentous structures such as hollow nanotubes or densely packed fibers that have all the properties of an ideal sustainedrelease formulation $[2,4]$. Firstly, they only contain the densely packed peptide (commonly as an acetate salt) and water, which enable very small injection volumes even for high doses. Secondly, they form highly organized and stable structures. The active drug, i.e., the peptide, is very stable in these structures and is released in a controlled manner over an extended period of time. This stability also enables sustainable supply and storage of prefilled, ready-to-inject syringes. Furthermore, release of the active drug from the depot can provide a rapid response [2].

Lanreotide is an analog of human somatostatin, and lanreotide depot (Somatuline Depot; Ipsen Pharma SAS, Paris, France) is the first marketed sustained-release formulation produced via peptide self-assembly. Lanreotide depot has been used by patients with acromegaly for more than 13 years [5] and has recently been approved in the USA for the treatment of patients with unresectable, well- or moderately differentiated, locally advanced, or metastatic gastroenteropancreatic neuroendocrine tumors (GEP-NETs) to improve progression-free survival (PFS) [6]. Lanreotide depot has also been approved in Europe since 2003 for symptom control in carcinoid syndrome associated with GEP-NETs [5].

NETs are a group of malignancies that arise from the secretory cells of the neuroendocrine system. NETs can develop in virtually any organ but are predominantly found in the gastrointestinal system, particularly the pancreas and gastrointestinal tract [7]. GEP-NETs are rare; however, the ageadjusted incidence of GEP-NETs has been steadily increasing, with a 3.6-fold increase observed between 1973 and 2007 [7-9]. Improved disease awareness and improved diagnostic imaging technologies [10] may play a role in the apparent increase in incidence [7]. Based on the most updated data from the Surveillance, Epidemiology and End Results program of the National Cancer Institute, the incidence of GEP-NETs was 3.65/100,000 individuals per year between 2003 and 2007 [9].

While indolent in nature, advanced well-differentiated NETs remain incurable with overall poor prognosis, and only a few treatment options can effectively inhibit their growth [11-13]. Goals of therapy in managing patients with GEPNETs are twofold: symptoms and tumor control. The majority of NETs are nonfunctioning, i.e., they do not secrete excess hormones that cause clinical symptoms. This lack of symptoms in nonfunctioning NETs may delay diagnosis until after the disease has advanced and metastasized, when patients will exhibit symptoms relating to their bulky/advanced disease burden. Conversely, functioning NETs are characterized by excessive hormone secretion resulting in distinct clinical syndromes, depending on the hormone secreted. A common example is the carcinoid syndrome, which is caused by systemic release of serotonin and tachykinins by gastrointestinal NETs and characterized by severe gastrointestinal symptoms including flushing and diarrhea [11, 13]. Functioning and nonfunctioning NETs are histologically indistinguishable as they are clinically defined/classified, and goals of treatment remain the same - controlling of tumor and controlling of symptoms (from hormone over-secretion or from bulky tumor burden).

In most patients with advanced or metastatic GEP-NETs, curative surgery may not be an option, and systemic management is an important part of clinical care. Somatostatin analogs (SSAs) play a prominent role in systemic management of GEP-NETs. GEP-NETs frequently overexpress receptors for the inhibitory hormone somatostatin, and SSAs have been shown to be effective in controlling symptoms of hormone hypersecretion in a number of functioning GEP-NETs [7, 13]. Furthermore, recent studies have shown that SSAs also have antiproliferative effects in GEP-NETs [12, 15].

This review discusses how the formulation of lanreotide depot provides potential advantages to both patients and health care providers, as well as the most recent clinical evidence demonstrating the safety and efficacy of lanreotide depot in the control of tumor growth and hormonal symptoms in GEP-NETs.

\section{Lanreotide Depot Formulation}

The first long-acting formulation of lanreotide (Somatuline LA, Ipsen Pharma) comprised a polymeric microsphere system and was intended for dosing every 7, 10, or 14 days. Polymeric microsphere-based formulation systems have been shown to be effective and have also been used in other longacting SSAs. However, microsphere-based formulations have inherent disadvantages, including the need for reconstitution before injection, a complex manufacturing process requiring the use of organic solvents that are often toxic, potential burst release of the drug, the generation of acidic metabolites during the polymer degradation process, and possible degradation of the peptide [3, 16-18]. Moreover, microsphere-based formulations often necessitate potentially painful intramuscular injections as well as large injection volumes that can limit the amount of drug administered at a time and hence the length of drug exposure. Thus, an innovative approach was required to develop a new formulation for lanreotide with an improved pharmacokinetic (PK) profile and free of the aforementioned disadvantages.

Studies on lanreotide formulation led to a remarkable discovery that the peptide self-assembles into a gel under certain conditions. After extensive work led by Cherif-Cheikh and Valery et al. [19-28], the self-assembly process and liquid crystal structure of lanreotide depot formulation are now well characterized. The process starts with noncovalent peptide dimerization, formation, and growth of an open ribbon and ends with ribbon closure in the shape of a nanotube (Fig. 1a) [23]. In the nanotube, dimeric building blocks form $\beta$-sheet- 
Fig. 1 a Lanreotide is a cyclic octapeptide that can form noncovalently bonded dimers. The dimers constitute $\beta$-sheetrich filaments that are stabilized by hydrogen bonding. Twenty-six of the filaments make up the hollow nanotubes, which have a highly uniform diameter of $24 \mathrm{~nm}$. Depending on the lanreotide concentration, nanotubes in the semi-solid gel can be organized in a hexagonal lattice or form densely packed tube-within-a-tube structures (embedded nanotubes). Freezefracture electron micrographs of lanreotide in water $(14 \%, w / w)$, with the plane of fracture $\mathbf{b}$ perpendicular or $\mathbf{c}$ parallel to the direction of nanotubes. Insets are $2 \times$ enlarged. Scale bar $=100 \mathrm{~nm}$ [25-27]. b, c Reprinted from [25] a
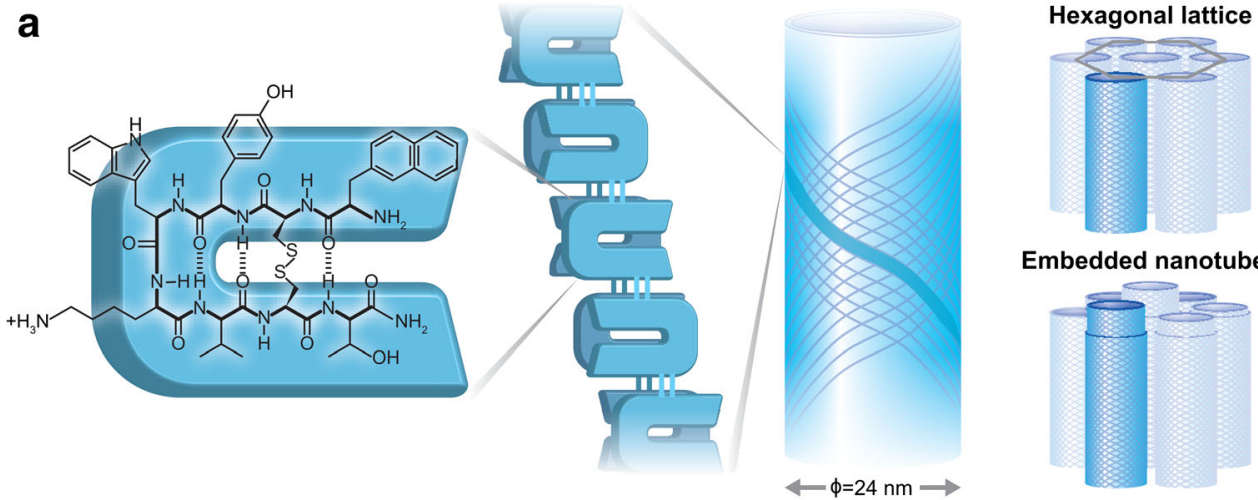

Embedded nanotubes

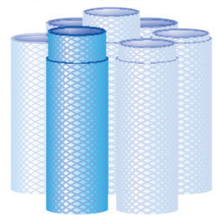

$\phi=24 \mathrm{~nm} \longrightarrow$

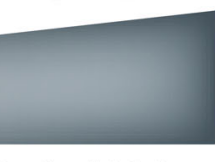

Lanreotide

$\beta$-sheet rich filaments

Nanotube

Semi-solid Gel

b

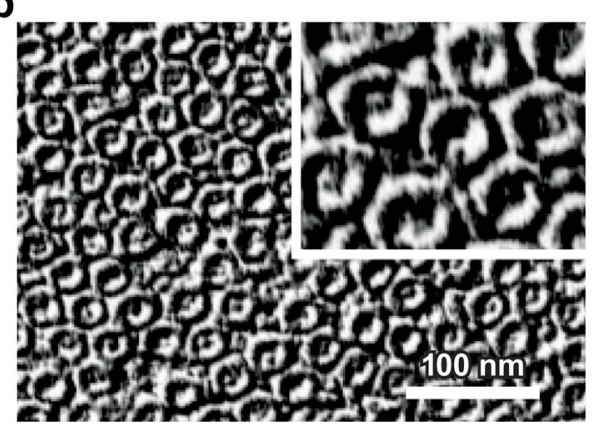

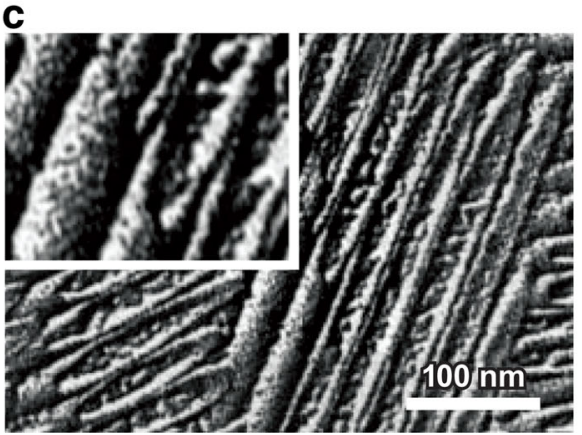

rich filaments that constitute the hollow nanotubes with a highly uniform diameter and wall thickness. The structure of the semi-solid gel results from a very dense packing of the nanotubes. At lanreotide concentrations of up to 10 to $15 \%$ $(w / w)$, the semi-solid gel is primarily composed of an organized hexagonal lattice of nanotubes (Fig. 1b, c), whereas the nanotubes start to form a densely packed tube-within-a-tube structure ("embedded nanotubes") at higher lanreotide concentrations (Fig. 1a) [25, 26]. The nanotubes are stabilized by noncovalent forces including hydrogen bonding between dimeric subunits, hydrophobic interactions, and $\pi-\pi$ stacking of the aromatic side chains within the filaments (Fig.1a) [27]. These interactions can form and be maintained in water, without the need for any other substance or excipient. An understanding of the biophysical forces involved in nanotube assembly will help future development of self-assemblybased peptide formulations. Most importantly, in vitro experiments have shown that the nanotube assembly is completely reversible. At low lanreotide concentration, the nanotubes slowly disassemble, releasing the active drug $[19,23]$. The slow and controlled drug release that starts immediately upon transfer into a dilute solution renders the nanotube formulation an ideal candidate for fast-acting and sustained delivery of lanreotide.
In vivo, lanreotide is believed to form a depot at the injection site, based on the interaction of the formulation with physiological fluids. In vivo release of lanreotide is governed by the intrinsic properties of self-assembly of the peptide itself and is most likely mediated by passive diffusion of lanreotide from the depot into the surrounding tissues, followed by the absorption to the bloodstream (Fig. 2a) [6, 29]. Indeed, PK studies confirmed the favorable PK profile expected based on the previous data. When a small volume of concentrated lanreotide self-assembly formulation $(120 \mathrm{mg}, 0.5 \mathrm{~mL})$ was injected subcutaneously in healthy volunteers, it formed a rapid-acting depot (Fig. 2a), with significant lanreotide concentrations reached within the first day as demonstrated by the short time to reach peak serum concentration $\left(T_{\max }, 7 \mathrm{~h}\right)$. The subcutaneous depot provided a controlled, sustained release of the drug over extended periods of time with a low peak serum concentration $\left(C_{\max }, 6.79 \mathrm{ng} / \mathrm{mL}\right)$ and long terminal half-life (30.1 days). The mean serum concentration 4 weeks after injection was $1.69 \mathrm{ng} / \mathrm{mL}$ (Fig. 2b, c) [5, 6]. PK properties were similar in patients with acromegaly, and a linear PK profile between the 60 - and 120-mg doses was observed [30, 31]. In patients with acromegaly, the mean (SD) steady-state trough serum lanreotide concentrations in patients receiving lanreotide depot every 4 weeks were $1.8(0.3), 2.5(0.9)$, and 
a
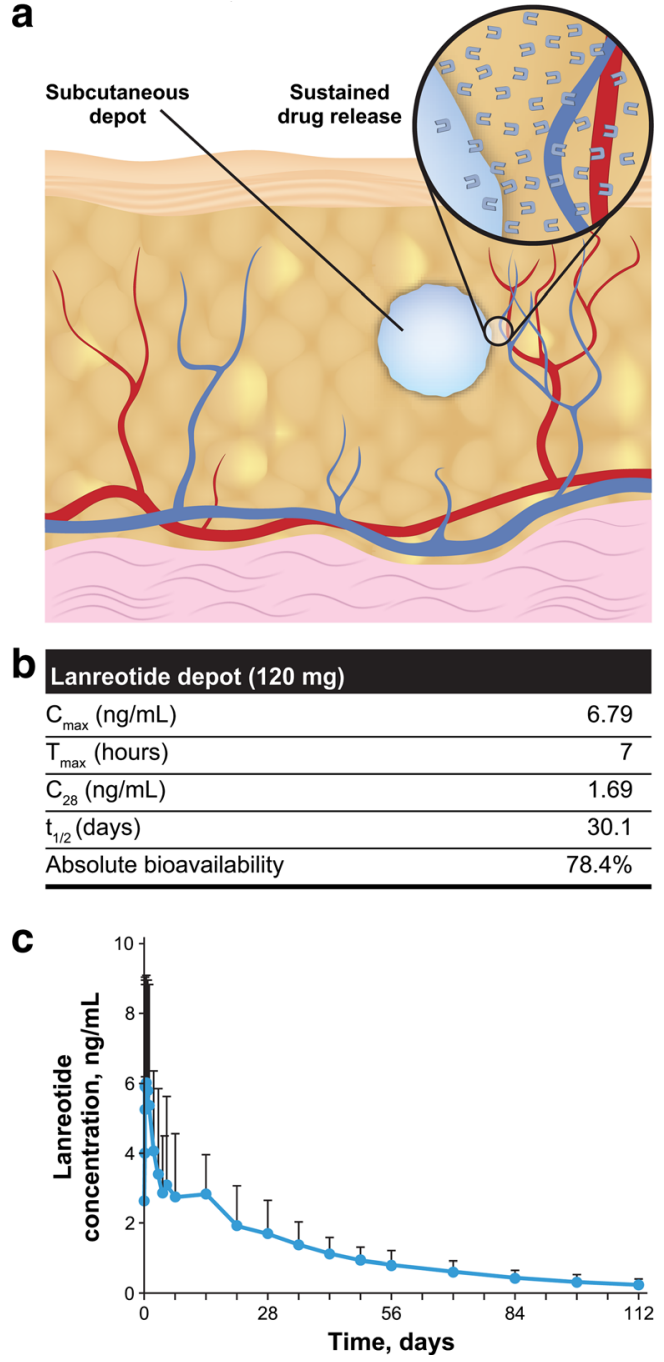

Fig. 2 a Schematic view of subcutaneous depot and sustained drug release. Inset shows diffusion of active drug to the adjacent capillaries. b Pharmacokinetic properties and concentration-time curve of lanreotide depot after a single 120-mg injection in healthy volunteers $[5,6] . C_{28}$ mean serum concentration 28 days after injection, $C_{\max }$ peak serum concentration, $T_{\max }$ time to reach $C_{\max }, t_{1 / 2}$ apparent terminal elimination half-life

$3.8(1.0) \mathrm{ng} / \mathrm{mL}$ at $60-\mathrm{mg}, 90-\mathrm{mg}$, and $120-\mathrm{mg}$ doses, respectively $[6,30]$. In patients with GEP-NETs who received a 120 -mg dose of lanreotide depot every 4 weeks, steadystate concentrations were reached after four to five injections, and the mean trough serum lanreotide concentrations at steady state were 5.3 to $8.6 \mathrm{ng} / \mathrm{mL}$ [6]. The PK properties of lanreotide depot formulation render it suitable for a 4week dosing interval [6].

The depot formulation also confers significant advantages in terms of administration, such as enabling sustained supply and storage of ready-to-use prefilled dosing devices, which eliminates the need for a multistep reconstitution process. It also allows small-volume subcutaneous administration of the drug [6]. Moreover, a new injection device for lanreotide, which has recently been approved in the USA, provides additional safety advantages including a rigid needle cap, automatic needle guard to prevent needlestick injuries, and a fully transparent syringe to help confirm successful delivery [32].

The potential advantages of lanreotide depot formulation and the new injection device were assessed in a quantitative opinion study conducted with 77 nurses in Europe and the USA; all of whom had previous experience with SSAs [32]. To evaluate the administration of two long-acting SSAs in clinical practice (lanreotide depot [subcutaneous] and octreotide LAR [intramuscular; Sandostatin LAR; Novartis, Basel, Switzerland]), the participants were asked to score both drugs in terms of 16 administration-related attributes, and lanreotide depot received a significantly higher overall preference score $(114$ vs $70, P<0.01)$ (Table 1$)$. The only attribute for which the drugs received a similar preference score was the sturdiness of the plunger (Table 1). Confidence that a full dose had been delivered was the most important attribute according to the nurses. Indeed, improved reliability and consistent delivery of accurate drug doses may have a positive effect on observed drug efficacy, particularly in patients who receive care in a community setting. Moreover, easy and quick administration, as well as the low risk of clogging, could help conserve time and resources [33].

Subcutaneous delivery of lanreotide depot, which was received favorably in the aforementioned nurse opinion study (Table 1) [32], may offer important advantages in ease and accuracy of administration. For instance, intramuscular injections can be associated with significant risk of unintended subcutaneous placement [34], particularly in obese patients because of the thick layer of adipose limiting access to muscle tissue or in cachectic patients who have lost significant muscle mass [35]. The high rate of improper intramuscular injections may reduce the effectiveness of the medicine in a community setting and increases the potential risk of tissue damage [34, 35]. Moreover, intramuscular SSA injections are more commonly associated with injection-site pain than are subcutaneous SSA injections [36].

Further studies are needed to confirm the findings of the nurse opinion study in real-world practice and assess the potential of lanreotide depot formulation to meet expectations of both health care providers and patients.

\section{Efficacy and Safety of Lanreotide Depot in NETs}

For decades, SSAs have played a significant role in the management of hormonal symptoms in metastatic GEP-NETs [5, 37]. Lanreotide is approved for the treatment of carcinoid syndrome in more than 50 countries but not yet in the USA. In light of recent clinical evidence including the landmark study CLARINET, lanreotide depot is now also approved by the US Food and Drug Administration (FDA) for the 
Table 1 Nurse's preference with self-assembly formulation [32]

\begin{tabular}{|c|c|c|c|}
\hline \multirow[t]{2}{*}{ Attribute } & \multirow{2}{*}{$\begin{array}{l}\text { Mean relative importance } \\
\text { score } \\
\text { (scale of } 1-10)\end{array}$} & \multicolumn{2}{|c|}{$\begin{array}{l}\text { Mean preference score } \\
\text { (scale of } 1-10 \text { ) }\end{array}$} \\
\hline & & $\begin{array}{l}\text { Octreotide } \\
\text { LAR }\end{array}$ & $\begin{array}{l}\text { Lanreotide } \\
\text { depot }\end{array}$ \\
\hline $\begin{array}{l}\text { Confidence that a full dose has been } \\
\text { delivered }\end{array}$ & 9.5 & 6.8 & 9.5 \\
\hline High product efficacy & 9.4 & 7.5 & 8.6 \\
\hline Good safety & 9.4 & 5.8 & 8.9 \\
\hline Low risk of clogging & 8.8 & 3.7 & 9.4 \\
\hline Easy/convenient preparation and injection & 8.8 & 3.8 & 9.4 \\
\hline Easy to teach & 8.3 & 4.4 & 9.1 \\
\hline Low risk of needlestick injuries & 8.2 & 4.9 & 8.8 \\
\hline Prefilled device & 8.1 & 3.1 & 9.5 \\
\hline Calm environment for patient & 7.9 & 6.4 & 7.9 \\
\hline $\begin{array}{l}\text { Fast administration (preparation \& } \\
\text { injection) }\end{array}$ & 7.9 & 3.7 & 9.3 \\
\hline Sturdy plunger & 7.9 & 7.7 & 7.5 \\
\hline Transparent device & 7.8 & 7.8 & 9.2 \\
\hline Comfortable device to hold & 7.7 & 6.3 & 7.6 \\
\hline Short/thin needle & 7.6 & 3.8 & 7.1 \\
\hline Depth of injection (IM vs deep SC) & 7.2 & 5.6 & 8.1 \\
\hline Self-injection possible & 6.8 & 3.2 & 8.4 \\
\hline Overall preference score & & 70 & 114 \\
\hline
\end{tabular}

Nurses rated a predefined list of attributes on a scale of 1 to $10(1=$ not important/not preferred, $10=$ most important/most preferred). Lanreotide depot (new device) received higher preference scores in all attributes except for the sturdiness of the plunger. Overall preference score, which included scores in all 16 attributes weighted by relative importance, was significantly higher with lanreotide depot $(P<0.01)$

$I M$ intramuscular, $S C$ subcutaneous treatment of patients with unresectable, well- or moderately differentiated, locally advanced GEP-NETs to improve PFS [6]. In this section, three recent clinical trials evaluating the efficacy and safety of lanreotide depot in the treatment of GEP-NETs will be discussed.

\section{CLARINET: Antiproliferative Effect in Advanced GEP-NETs}

CLARINET was a 96-week, randomized, double-blind, placebo-controlled, parallel-group, international, phase 3 study in 204 patients with advanced, well- or moderately differentiated, nonfunctioning, metastatic, and/or locally advanced, unresectable, grade 1 or $2(\mathrm{Ki}-67<10 \%)$ GEP-NETs.

The primary end point was PFS, defined as the time to disease progression or death. Secondary end points included overall survival, quality of life, and safety [12].

CLARINET demonstrated that lanreotide depot $120 \mathrm{mg}$ administered subcutaneously once every 4 weeks significantly improved PFS in patients with advanced, well- or moderately differentiated, somatostatin receptor-positive GEP-NETs, regardless of hepatic tumor volume [12]. In patients who received

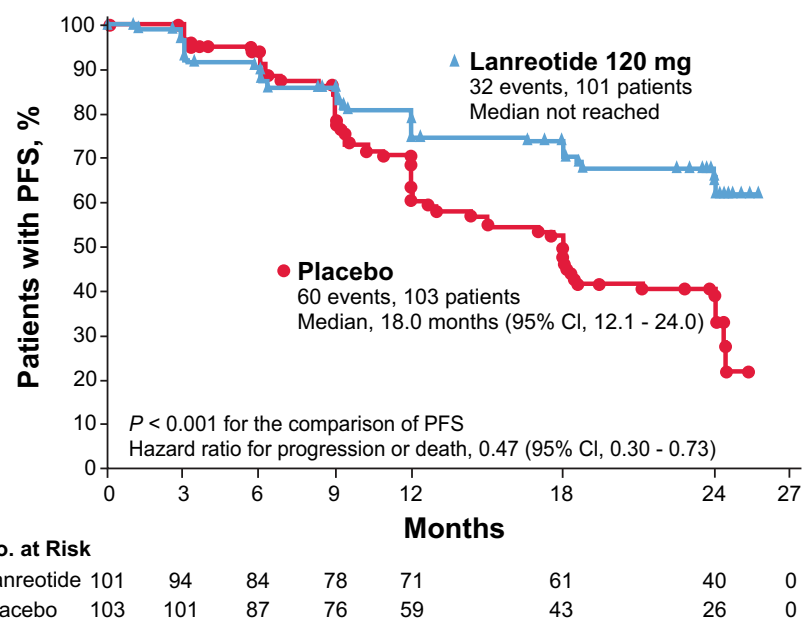

Fig. 3 Estimates of PFS among patients who received lanreotide depot $(120 \mathrm{mg}$ ) or placebo in the 96-week, placebo-controlled CLARINET core study. Kaplan-Meier curves were compared using a stratified log-rank test. Stratification was based on the presence or absence of tumor progression at baseline and the receipt or nonreceipt of previous therapy. The hazard ratio was derived from a Cox proportional hazards model with terms for study treatment, the presence or absence of tumor progression at baseline, and the receipt or nonreceipt of previous therapy [12]. CI confidence interval, PFS progression-free survival. From [12] (reprinted with permission from Massachusetts Medical Society) 
Table 2 Secondary efficacy end points in CLARINET core study (intent-to-treat population) [12]

\begin{tabular}{|c|c|c|c|}
\hline End point & $\begin{array}{l}\text { Lanreotide depot } \\
(n=101)\end{array}$ & $\begin{array}{l}\text { Placebo } \\
(n=103)\end{array}$ & $P$ value \\
\hline \multicolumn{4}{|l|}{ Patients alive without disease progression ${ }^{\mathrm{a}}$} \\
\hline Week 48 & $66 \%$ & $49 \%$ & $<0.05$ \\
\hline Week 96 & $52 \%$ & $25 \%$ & $<0.001$ \\
\hline $\begin{array}{l}\text { Median time to tumor progression }(95 \% \mathrm{CI}) \text {, } \\
\text { months } \mathrm{b}^{\mathrm{b}}\end{array}$ & Not reached & $18.0(12.1$ to 24.0$)$ & $<0.001^{\mathrm{c}}$ \\
\hline $\begin{array}{l}\text { Patients with } \geq 50 \% \text { reduction in level of } \\
\text { chromogranin A from baseline to last post-baseline } \\
\text { level available }\end{array}$ & $42 \%$ & $5 \%$ & $<0.001$ \\
\hline
\end{tabular}

CI confidence interval

${ }^{\text {a }}$ The measures of progression-free survival shown here are different from the primary end point of CLARINET in that they are measurements at discrete time points and with patients counted as having treatment success or failure at each assessment

${ }^{\mathrm{b}}$ There were 30 events in the lanreotide group and 58 events in the placebo group

${ }^{\mathrm{c}}$ The between-group difference was analyzed with the use of the log-rank test

${ }^{\mathrm{d}}$ Data are shown for the subgroup of patients with a baseline chromogranin A level higher than the upper limit of the normal range $(98.1 \mu \mathrm{g} / \mathrm{L})$ who also had evaluable data after baseline (64 patients in each group). Calculations were made with the use of a logistic-regression model with terms for study treatment and receipt or nonreceipt of previous therapy lanreotide depot, median PFS was not reached and was 18.0 months in patients who received placebo $(P<0.001)$. The risk of disease progression or death within 96 weeks of first dose was significantly reduced with lanreotide (hazard ratio
0.47; $95 \%$ confidence interval [CI], 0.30-0.73) (Fig. 3) [12]. At week 96, estimated rates of PFS were $65 \%$ in the lanreotide depot group and $33 \%$ in the placebo group. At both weeks 48 and 96, the percentage of patients alive without disease
Table 3 Summary of AEs in CLARINET core study (safety population) [12]

\begin{tabular}{|c|c|c|}
\hline \multirow[t]{2}{*}{ Event } & $\begin{array}{l}\text { Lanreotide depot } \\
(n=101)\end{array}$ & $\begin{array}{l}\text { Placebo } \\
(n=103)\end{array}$ \\
\hline & \multicolumn{2}{|c|}{ No. of patients, $\%$} \\
\hline Any AE & $89(88)$ & $93(90)$ \\
\hline Any $\mathrm{AE}$ related to study treatment & $50(50)$ & $29(28)$ \\
\hline \multicolumn{3}{|l|}{ Any $\mathrm{AE}$ according to intensity ${ }^{\mathrm{a}}$} \\
\hline Severe & $26(26)$ & $32(31)$ \\
\hline Moderate & $44(44)$ & $44(43)$ \\
\hline Mild & $17(17)$ & $17(17)$ \\
\hline \multicolumn{3}{|c|}{ Study treatment-related AEs in $\geq 5 \%$ of patients } \\
\hline Diarrhea & $26(26)$ & $9(9)$ \\
\hline Abdominal pain & $14(14)$ & $2(2)$ \\
\hline Cholelithiasis & $10(10)$ & $3(3)$ \\
\hline Flatulence & $8(8)$ & $5(5)$ \\
\hline Injection-site pain & $7(7)$ & $3(3)$ \\
\hline Nausea & $7(7)$ & $2(2)$ \\
\hline Vomiting & $7(7)$ & 0 \\
\hline Headache & $5(5)$ & $2(2)$ \\
\hline Lethargy & $5(5)$ & $1(1)$ \\
\hline Hyperglycemia & $5(5)$ & 0 \\
\hline Decreased level of pancreatic enzymes & $5(5)$ & 0 \\
\hline
\end{tabular}

AEs were defined according to the Medical Dictionary for Regulatory Activities, version 16.0 $A E$ adverse event

${ }^{\text {a }}$ For patients with multiple AEs, events with the maximum intensity are shown; data are missing for two patients in the lanreotide group 
progression was higher in the lanreotide depot group (Table 2) [12]. The PFS benefits were observed in almost all predefined subgroups regardless of tumor origin (midgut, pancreas, other), tumor grade (grade 1 or 2), and hepatic tumor volume ( $>25$ or $\leq 25 \%$ ) [12]. A significant effect of lanreotide depot on overall survival was not evident, possibly due to relatively long life expectancy in patients with slow-growing tumors [38]. In addition, crossovers from placebo to lanreotide depot and treatments received after progression may have complicated the analysis [12]. Among patients with higher-than-normal baseline levels of NET biomarker chromogranin A, a more than or equal to $50 \%$ reduction in chromogranin A levels was achieved in $42 \%$ of patients treated with lanreotide depot compared with only $5 \%$ in the placebo group (Table 2) [12].

In accordance with the accumulated data demonstrating safety of lanreotide depot in other indications including long-term experience in patients with functioning NETs [6, 39], lanreotide depot was generally well tolerated in CLARINET [12]. Despite the relatively long median exposure time (24.0 months), lanreotide depot treatment did not increase the proportion of patients who had adverse events (AEs) $(88 \%$ compared with $90 \%$ with placebo), and most of the AEs experienced in both groups were mild or moderate. The most common study-related AEs were gastrointestinal related and included diarrhea (26\%) and abdominal pain (14\%) (Table 3) [12]. In addition, lanreotide depot treatment did not cause deterioration in the quality of life as assessed by the European Organization for Research and Treatment of Cancer Quality of Life Questionnaire-Core 30 (EORTC-QLQ-C30) global health status score [12].

A total of 88 patients from the core study (41 from the lanreotide group, 47 from the placebo group) participated in the open-label extension of CLARINET. A median PFS of 32.8 months was reached for the lanreotide group during the extension. No new safety concerns were identified [40].

A study in 85 patients with low-grade (grade 1), midgut NETs demonstrated that octreotide LAR (30-mg intramuscular injection, every 4 weeks) prolongs time to tumor progression in the studied patient population (PROMID study) [15]. This study supports the body of literature regarding the activity of SSAs as antiproliferative agents in NETs. However, octreotide LAR is not FDA-approved as an antiproliferative agent to improve PFS in GEP-NETs [37].

Although CLARINET included only patients with nonfunctioning tumors, earlier open-label studies of lanreotide depot have suggested activity in all patients with GEP-NETs regardless of tumor-functioning status [41, 42]. The presence of a functional hormone secretion syndrome is not relevant in terms of antiproliferative treatments, and tumor grade as well as somatostatin receptor expression status are among the most important factors that determine response to SSAs in GEPNETs [13, 14]. Accordingly, lanreotide depot is approved in the USA for the treatment of both functioning and nonfunctioning GEP-NETs [6].
In summary, CLARINET showed that lanreotide depot prolonged PFS in patients with advanced, grade 1 to 2, somatostatin receptor-positive GEP-NETs regardless of hepatic tumor volume.

\section{ELECT and SYMNET Studies: Symptom Control in Carcinoid Syndrome}

Two recent studies have evaluated the efficacy of lanreotide depot in control of hormonal symptoms associated with carcinoid syndrome: ELECT and SYMNET [43, 44]. The phase 3 ELECT study included a 16-week, double-blind, placebocontrolled phase and a 32-week open-label extension phase and was conducted in SSA-naive (44\%) and octreotideresponsive $(56 \%)$ patients $(n=115)$. Patients were randomized to receive subcutaneous lanreotide depot $120 \mathrm{mg}$ every 4 weeks or placebo. Because assessment of symptom control in carcinoid syndrome has always been a difficult study outcome to evaluate, a novel primary end point was used: mean
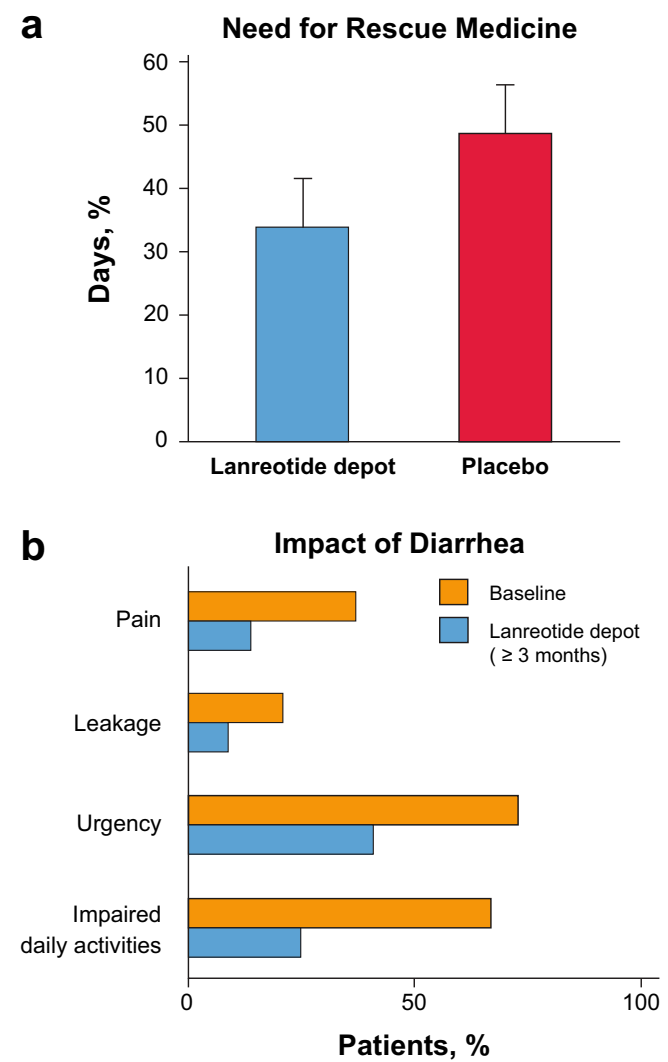

Fig. 4 a In the ELECT study, mean percentage of days with rescue medication use was statistically lower with lanreotide depot (34 [95\% CI, 25-42]) vs placebo (49 [95\% CI, 40-57]) $(P=0.02)$. Error bars denote $95 \%$ CI. b In the SYMNET study, the impact of diarrhea on patients was reduced with lanreotide depot ( $\geq 3.0$ months) treatment. There were statistically significant reductions in the numbers of patients with diarrhea-related pain (37 vs $14 \%$ ), leakage (21 vs $9 \%$ ), and urgency (73 vs $41 \%$ ) (McNemar paired tests, $P<0.001)$. Fewer patients had impaired daily activities due to diarrhea during treatment $(25 \%)$ than before $(67 \%)[43,44]$. CI confidence interval 
percentage of days requiring rescue medication (MPDR). Patients were allowed to use a rescue medication (shortacting octreotide; Sandostatin; Novartis, Basel, Switzerland) to control breakthrough symptoms throughout the study, and MPDR was significantly lower in lanreotide depot vs placebo arms, at 34 vs $49 \%(P=0.017$, Fig. 4a). The primary end point was met, and this represented a clinically significant difference of approximately 5 days less use of a rescue medication on the lanreotide depot arm. Moreover, complete or partial success, defined as $\leq 3$ days use of rescue medication per week, was achieved significantly more commonly with lanreotide depot compared with the placebo group (odds ratio 2.4; $95 \%$ CI, 1.1-5.3) [43]. Treatment-emergent AEs were observed in $26 \%$ of patients in the lanreotide depot group compared with $19 \%$ with placebo. Most AEs were not serious, and only one patient from each group discontinued the treatment due to AEs. The most frequent AEs were gastrointestinal [43].

SYMNET was a large ( $n=273)$, multinational, cross-sectional, observation study to evaluate patient satisfaction with symptom control during lanreotide depot treatment in patients with GEP-NETs who experienced carcinoid syndrome-related diarrhea [44]. At routine clinical visits, patients who had been receiving lanreotide depot for at least 3.0 months completed questionnaires. Satisfaction with diarrhea control was the primary end point. The majority of patients $(79 \%)$ reported improved diarrhea control with lanreotide depot, and most patients $(76 \%)$ were completely or rather satisfied with this effect. The satisfaction regarding the control of flushing was similarly high $(73 \%)$. Compared with baseline, a clinically significant decrease in median daily stool frequency (from 4 to 2 ) was observed. The overall impact of diarrhea on patients was also significantly lower than baseline, as evidenced by reduced pain, leakage, urgency, and impact on daily activities (Fig. 4b). The ELECT and SYMNET studies confirmed that lanreotide may help patients sustain good control of carcinoid syndrome symptoms.

\section{Conclusions}

Interest in peptide-based antineoplastic drugs has been increasing; however, administration of these drugs has historically posed significant challenges in terms of sustaining a therapeutic dose over a long period of time. Peptide selfassembly-based formulations provide an innovative solution to these challenges.

Lanreotide depot is a chemically distinct SSA and the first marketed drug with a self-assembly-based sustained-release activity. Extensive research on the lanreotide peptide selfassembly process provides insights that could help future development of self-assembly-based formulations for therapeutic peptides or the bottom-up design of new peptide families that self-assemble into complex architectures with desired properties.

SSAs have been used for the treatment of symptoms associated with carcinoid syndrome, and now, based on evidence from a phase 3 study, lanreotide depot is the first, and currently the only, SSA that is approved in the USA for the treatment of metastatic GEP-NETs to improve PFS. Moreover, lanreotide depot's formulation and method of delivery provide the potential to improve quality of life and tumor management in patients with GEP-NETs.

Acknowledgments This study was supported by Ipsen Biopharmaceuticals, Inc., Basking Ridge, NJ. S. Tanik, PhD, of ClinicalThinking, Hamilton, NJ and Debika Chatterjea, PhD, of MedVal Scientific Information Services, Skillman, NJ provided medical writing assistance which was funded by Ipsen.

\section{Compliance with Ethical Standards}

Conflict of Interest EMW: consulting or advisory role-Celgene, Ipsen, Novartis, Pfizer; research funding-Ipsen (Inst); Novartis (Inst); Pfizer (Inst)

AM: employee of Ipsen

CC: employee of Ipsen

AL: employee of Ipsen

LB: employee of Ipsen

JR: employee of Ipsen

ATP: received honoraria-Novartis, Celgene, Ipsen, Lilly, Genentech; consulting or advisory role-Novartis; speakers bureauNovartis, Celgene, Ipsen, Lilly, Genentech

Open Access This article is distributed under the terms of the Creative Commons Attribution 4.0 International License (http:// creativecommons.org/licenses/by/4.0/), which permits unrestricted use, distribution, and reproduction in any medium, provided you give appropriate credit to the original author(s) and the source, provide a link to the Creative Commons license, and indicate if changes were made.

\section{References}

1. Thundimadathil J. Cancer treatment using peptides: current therapies and future prospects. J Amino Acids. 2012;2012:967347.

2. Maji SK, Schubert D, Rivier C, et al. Amyloid as a depot for the formulation of long-acting drugs. PLoS Biol. 2008;6:e17.

3. Lewis AL, Illum L. Formulation strategies for sustained release of proteins. Ther Deliv. 2010;1:457-79.

4. Dobson CM. The structural basis of protein folding and its links with human disease. Philos Trans R Soc Lond Ser B Biol Sci. 2001;356:133-45.

5. Somatuline Autogel [SPC]. Somatuline Autogel, solution for injection in a prefilled syringe [summary of product characteristics]. Paris: Ipsen Ltd; 2013.

6. Somatuline Depot [package insert]. Basking Ridge, NJ: Ipsen Biopharmaceuticals, Inc; 2014.

7. Cives M, Strosberg J. An update on gastroenteropancreatic neuroendocrine tumors. Oncology (Williston Park). 2014;28:201359. 
8. Fraenkel M, Kim M, Faggiano A, et al. Incidence of gastroenteropancreatic neuroendocrine tumours: a systematic review of the literature. Endocr Relat Cancer. 2014;21:R153-63.

9. Lawrence B, Gustafsson BI, Chan A, et al. The epidemiology of gastroenteropancreatic neuroendocrine tumors. Endocrinol Metab Clin N Am. 2011;40:1-18 .vii

10. Lawrence B, Gustafsson BI, Kidd M, et al. The clinical relevance of chromogranin A as a biomarker for gastroenteropancreatic neuroendocrine tumors. Endocrinol Metab Clin N Am. 2011;40:111-34 viii

11. Oberg KE. The management of neuroendocrine tumours: current and future medical therapy options. Clin Oncol (R Coll Radiol). 2012;24:282-93.

12. Caplin ME, Pavel M, Cwikla JB, et al. Lanreotide in metastatic enteropancreatic neuroendocrine tumors. N Engl J Med. 2014;371:224-33.

13. Wolin EM. The expanding role of somatostatin analogs in the management of neuroendocrine tumors. Gastrointest Cancer Res. 2012;5:161-8.

14. Modlin IM, Moss SF, Gustafsson BI, et al. The archaic distinction between functioning and nonfunctioning neuroendocrine neoplasms is no longer clinically relevant. Langenbeck's Arch Surg. 2011;396:1145-56.

15. Rinke A, Muller HH, Schade-Brittinger C, et al. Placebo-controlled, double-blind, prospective, randomized study on the effect of octreotide LAR in the control of tumor growth in patients with metastatic neuroendocrine midgut tumors: a report from the PROMID study group. J Clin Oncol. 2009;27:4656-63.

16. Plourde F, Motulsky A, Couffin-Hoarau AC, et al. First report on the efficacy of 1-alanine-based in situ-forming implants for the longterm parenteral delivery of drugs. J Control Release. 2005;108: 433-41.

17. Siepmann J, Faisant N, Akiki J, et al. Effect of the size of biodegradable microparticles on drug release: experiment and theory. $\mathrm{J}$ Control Release. 2004;96:123-34.

18. Estey T, Kang J, Schwendeman SP, et al. BSA degradation under acidic conditions: a model for protein instability during release from PLGA delivery systems. J Pharm Sci. 2006;95:1626-39.

19. Gobeaux F, Fay N, Tarabout C, et al. Structural role of counterions adsorbed on self-assembled peptide nanotubes. J Am Chem Soc. 2012;134:723-33.

20. Gobeaux F, Fay N, Tarabout C, et al. Experimental observation of double-walled peptide nanotubes and monodispersity modeling of the number of walls. Langmuir. 2013;29:2739-45.

21. Pandit A, Fay N, Bordes L, et al. Self-assembly of the octapeptide lanreotide and lanreotide-based derivatives: the role of the aromatic residues. J Pept Sci. 2008;14:66-75.

22. Pouget E, Dujardin E, Cavalier A, et al. Hierarchical architectures by synergy between dynamical template self-assembly and biomineralization. Nat Mater. 2007;6:434-9.

23. Pouget E, Fay N, Dujardin E, et al. Elucidation of the self-assembly pathway of lanreotide octapeptide into beta-sheet nanotubes: role of two stable intermediates. J Am Chem Soc. 2010;132:4230-41.

24. Tarabout C, Roux S, Gobeaux F, et al. Control of peptide nanotube diameter by chemical modifications of an aromatic residue involved in a single close contact. Proc Natl Acad Sci U S A. 2011;108:767984.

25. Valery $\mathrm{C}$, Paternostre $\mathrm{M}$, Robert $\mathrm{B}$, et al. Biomimetic organization: octapeptide self-assembly into nanotubes of viral capsid-like dimension. Proc Natl Acad Sci U S A. 2003;100:10258-62.

26. Valery C, Artzner F, Robert B, et al. Self-association process of a peptide in solution: from beta-sheet filaments to large embedded nanotubes. Biophys J. 2004;86:2484-501.
27. Valery C, Pouget E, Pandit A, et al. Molecular origin of the selfassembly of lanreotide into nanotubes: a mutational approach. Biophys J. 2008;94:1782-95.

28. Cherif-Cheikh R, Bismuth F, Torres M, et al. Autogel: a new lanreotide prolonged release formulation. Proceed Int'l Symp Control Rel Bioact Mater. 1998;25:798.

29. Troconiz IF, Cendros JM, Peraire C, et al. Population pharmacokinetic analysis of lanreotide Autogel in healthy subjects: evidence for injection interval of up to 2 months. Clin Pharmacokinet. 2009;48:51-62.

30. Bronstein M, Musolino N, Jallad R, et al. Pharmacokinetic profile of lanreotide Autogel in patients with acromegaly after four deep subcutaneous injections of 60,90 or $120 \mathrm{mg}$ every 28 days. Clin Endocrinol. 2005;63:514-9.

31. Antonijoan RM, Barbanoj MJ, Cordero JA, et al. Pharmacokinetics of a new Autogel formulation of the somatostatin analogue lanreotide after a single subcutaneous dose in healthy volunteers. J Pharm Pharmacol. 2004;56:471-6.

32. Adelman DT, Burgess A, Davies PR. Evaluation of long-acting somatostatin analog injection devices by nurses: a quantitative study. Med Devices (Auckl). 2012;5:103-9.

33. Marty R, Roze S, Kurth H. Decision-tree model for health economic comparison of two long-acting somatostatin receptor ligand devices in France, Germany, and the UK. Med Devices (Auckl). 2012;5:39-44.

34. Boyd AE, DeFord LL, Mares JE, et al. Improving the success rate of gluteal intramuscular injections. Pancreas. 2013;42:878-82.

35. Palma S, Strohfus P. Are IM injections IM in obese and overweight females? A study in injection technique. Appl Nurs Res. 2013;26: e1-4.

36. Alexopoulou O, Abrams P, Verhelst J, et al. Efficacy and tolerability of lanreotide Autogel therapy in acromegalic patients previously treated with octreotide LAR. Eur J Endocrinol. 2004;151:317-24.

37. Sandostatin LAR [package insert]. East Hanover, NJ: Novartis Pharmaceuticals Corp; 2014.

38. Kulke MH, Siu LL, Tepper JE, et al. Future directions in the treatment of neuroendocrine tumors: consensus report of the National Cancer Institute Neuroendocrine Tumor clinical trials planning meeting. J Clin Oncol. 2011;29:934-43.

39. Khan MS, El-Khouly F, Davies P, et al. Long-term results of treatment of malignant carcinoid syndrome with prolonged release lanreotide (Somatuline Autogel). Aliment Pharmacol Ther. 2011;34:235-42.

40. Caplin ME, Pavel M, Ćwikła JB, et al. Anti-tumour effects of lanreotide for pancreatic and intestinal neuroendocrine tumours: the CLARINET open-label extension study. Endocr Relat Cancer. 2016:23:191-9.

41. Bajetta E, Procopio G, Catena L, et al. Lanreotide autogel every 6 weeks compared with lanreotide microparticles every 3 weeks in patients with well differentiated neuroendocrine tumors: a phase III study. Cancer. 2006;107:2474-81.

42. Martin-Richard M, Massuti B, Pineda E, et al. Antiproliferative effects of lanreotide autogel in patients with progressive, welldifferentiated neuroendocrine tumours: a Spanish, multicentre, open-label, single arm phase II study. BMC Cancer. 2013;13:427. doi:10.1186/1471-2407-13-427.

43. Vinik AI, Wolin EM, Liyanage N, et al. Evaluation of lanreotide depot/autogel efficacy and safety as a carcinoid syndrom treatment (ELECT): a randomized, double-blind, placebo-controlled trial. Endocr Pract. 2016 May 23. doi:10.4158/EP151172.OR.

44. Ruszniewski P, Valle JW, Lombard-Bohas C, et al. Patient-reported outcomes with lanreotide autogel/depot for carcinoid syndrome: an international observational study. Dig Liver Dis. 2016;48(5):552-8. 